Monatsschrift f. Geburtshülfe u. Gynäkologie 1930;86:I-IV

\title{
Contents, Vol. 86, 1930
}

Inhaltsverzeichnis.

Or $1 /$ sinalarbeiten. $\quad$ Seit $\theta$

Andreitschuk, T. S·, Biologische Behandlungsmethode der entzünd-

lichen Scheidenerkrankungen 48

Catel, Werner, Einfluß der Sterilisation der Frauenmilch auf den

Ernährungserfolg. (Zugleich Bemerkungen zu der Arbeit von Kayser

„Frauenmilch-Sammelstelle” in dieser Monatsschrift Band 84.) ... 36 - Erwiderung auf die

Ausführangen von M. E. Kayser auf Seite 41 . 46 Chatunzew, W. A., Ein Fall von primärem

Chorionepitheliom der Tube 60

Curschmann, Hans, Über postpartuale Magersucht 253

Derankowa, Elisabeth, Die Bestimmung der Frühschwangerschaft

mittels der Ninhydrin-Probe im Urin nach Dienst 8

Fraymann, S., Schläfrigkeit und Sehwangerschaft 15

Geller, Fr., und I. Krinke, Über die Dauererfolge der konservativen

Behandlung von Adnexentzündungen und ihre Leistungsfähigkeit im

Vergleich zur operativen Behandlung 288

G-las, Rudolf, Über Trypanblauspeicherung im Epithel der Gebär-

mutterschleimhaut der weißen Maus und Ratte 305

Goecke, H., siehe Haupt, W.

Gremme, A., Mereokranie und Exenzephalie415

Haupt, W., H. Goecke und J. Loevenich, Untersuchungen über

die Oxydationskraft des Blutes bei Eklamptischen nach Rodenacker 185 Hermstein, A., Über die partielle und sekundäre Blasenmolenbildung 268 Jagunow, S., Hämatologische Krisen bei den mit Radium behandelten

gynäkologischen Krebskranken 296

Joachimovits, Robert, Über typische und atypische Röntgenbefunde

bei Uterusmyom 65

Jaroschka, K., Über postoperative Diphtherieinfektionen 387

Kayser, Marie Elise, Erwiderung auf die Arbeit von Catel auf S. 3641 Keßler, Robert, Die

Therapie der Placenta praevia. (Unter Ver-

arbeitung des Materials der Kieler Universitäts-Frauenklinik vom

1. Januar 1923 bis 1. Januar 1930.) 175

Kir stein, Fr., Psychogene gynäkologische Beschwerden (Angstfolgen) 203 Klaften, E., Zur

Klinik und Anatomie der Granulosazelltumoren des

Eierstockes 392

Kohlhaas, Fritz, Ein Fall von malignem Cervixtumor bei einem

16 Monate alten Mädchen 58

Krinke, I., siehe Geller, Fr. Chr.

Krukenberg, Heinz, Zur Frage der Geburtsleitung beim engen 
Becken260

Loevenich, F., siehe Haupt, W.

Magyary, Kornel v., Die Veränderungen des Blutdrucks im Klimakterium 31

Mathias, Ernst, Bemerkenswerte Fälle aus der histologisch-gynäkologischen Praxis 218

Niedermeyer, Albert, Steißbeinresektion unter der Geburt .... 190

Peisachson, W., Ein Fall von zerviko-vaginaler Fistel 411

Sellheim, Hugo, Offenstehenbleiben der Scheide (Hiatus vaginae). 197 Sonnenfeld, Egon,

Kasuistische Beiträge zur Pathologie des Neu-

geborenen 210

Alle Rechte vorbehalten

Pierersche Hof'buchdruckereí Stephan Geibel \& Co., Altenburg, Thür.

YY I $\pi$ haltsverzeichnis.

Seite

Stein, Arthur, Die Anwendung der Gaseinblasung und Uterosalpingo-

graphie zur gynäkologischen Diagnose in den Vereinigten Staaten

von U.S.A. . 280

Vogt, E., Zur Behandlung der Meläna der Neugeborenen mit dem

Vitaminpräparat „Nateina” 422

Wagner, G. A., Geographische Verschiedenheiten beim Prolaps ... 1

Welscli, Kurt, Cliinovagin, ein neues Desinfektionsmittel für die Vagina 54

Berichie fiber ausiändische Literatur.

Die spanische geburtsliilfliche und gynäkologische Literatur 1929. Von

Dr. Victor Con ill-Barcelona 74

Übersicht über die geburtsliilfliche Literatur Amerikas im Jahre 1929.

Von J. P. Green hi 11, B.S., M.D., F.A.C.S. in Chikago 93

Die ungarische geburtshilflich-gynäkologische Literatur vom Jahre 1929.

Von Dr. Step han Liebmann-ßudapest 109

Fortschritte auf dem Gebiete der amerikanischen Gynäkologie während

des Jahres 1929. Brief aus Amerika. Von Dr. Arthur Stein-

New York 314

Vereins- und Literaturbeilage.

Original-Sitzungsberichte aus geburtshilflich-gynäkologischen Gesellschaften.

Gesellschaft für Geburtshilfe und Gynäkologie zu Berlin. Sitzungen vom

9. und 23. V. $1930 \quad 232$

- $\quad$ Sitzung vom 20. VI. $1930 \quad 325$

Gynäkologische Gesellschaft zu Breslau. Sitzung vom 13. V. 1930 . . 228

Münchener gynäkologische Gesellschaft. Sitzung vom 23. I. 1930 . . 134

Niederrheinisch-westfälische Gesellschaft für Gynäkologie und Geburts

hilfe. 92. Sitzung am 29. ill. 1930 zu Düsseldorf 138

Mittelrheinische Gesellschaft für Geburtshilfe und Gynäkologie. Sitzung

am 16. II. 1930 zu Frankfurt a. M 332

Nordwestdeutsche Gesellschaft für Geburtshilfe und Gynäkologie. Sitzung

am 10. V. 1930 in Bremen 343

Südostdeutsche und Mitteldeutsche Gesellschaft für Geburtshilfe und 
Gynäkologie. Sitzungen am 31. V. und 1. VI. 1930 zu Dresden . . 348 Geburtshilflichgynäkologische Gesellschaft in Wien. Sitzungen vom

13. V. und 10. VI. $1930 \quad 156$

Sitzung vom 24. VI. $1930 \quad 248$

VII. britischer Kongreß für Geburtshilfe und Gynäkologie unter Anwesenheit von Dr. Gibbon Fitzgibbon am 24. IV. 1929 in Dublin . 371

Döderleins-Tag 161

Klinische Vorlesung von Geheimrat August Martin im großen Hörsaal der Cliarité-Frauenklinik aniäßlich seines 60jährigen Doktorjubiläums Heinrich v. Peham. Ein Nachruf von J. Amreich 170

Hjalmar Forssner in memoriam 384

Friedrich Matthaei zum Gedächtnis ... 428

Buchbesprechu $\pi$ gen $160,249,379,426$

Personalien 174, 252

Sachregister zu Band 81-85 429

Namenregister zu Band 81-85 